\title{
Simulation of an All Optical Full Adder using Optical Switch
}

\author{
V. Khanaa ${ }^{1 *}$, K. P. Thooyamani ${ }^{2}$ and T. Saravanan ${ }^{3}$ \\ 'Dean, Information Technology Bharath University Chennai-600073, India; drvkannan62@yahoo.com \\ 2Professor of School of Computing, Bharath University, Chennai; thooyamani@hotmail.com \\ ${ }^{3}$ Professor \& Head, Department of ETC, Bharath University, Chennai-600073, India; saravanan.etc@bharathuniv.ac.in
}

\begin{abstract}
The optical communication system has many advantages at long distance communication. Semiconductor optical amplifier based Mach Zehnder Interferometer plays an important role in fast optical communication. In this work the proposed SOA based MZI switch is used for all optical logic and arithmetic operations. In this architecture the continuous wave laser source and control signal pass through the Mach Zehnder Interferometer. Control signal at this wave length is split at the coupler and more power passes through one arm. The input data signal is fed to the coupler, which splits the signal equally. The resultant signals then propagates simultaneously through the two arms of the interferometer. This architecture can be used to perform all optical processing of signals like half adder, full adder, de multiplexer etc. MZI switch is a very powerful technique to realize ultrafast switching. The physical mechanism behind the working of MZI switch is cross-phase modulation (XPM). Here the gain saturation induced by beam reduces carrier density inside one SOA, which in turn increases the refractive index only in the arm through which the light passes. As a result, an additional phase shift can be introduced on the CW beam because of the XPM, and the CW wave is directed towards the bar port during each one bit. Now it is clear that in the absence of control signal, the incoming signal (CW signal) exits through the cross port (lower channel) of MZI. In this case no light is present in the bar port. But in the presence of the control signal, the incoming signal exits through the bar port of the MZI.
\end{abstract}

Keywords: SOA, MZI, CW, OTA, Full Adder.

\section{Introduction}

An MZI switch is a very powerful technique to realize ultrafast switching. In this switch a SOA is inserted in each arm of an MZI. The pulsed signal at the wavelength is split at the first coupler such that more power passes through one arm. At the same time, the CW signal at the wavelength is split equally by this coupler and propagates simultaneously in the two arms. In the absence of the beam, the CW beam exits from the cross port (lower port in the figure). However, when both means are present simultaneously, all one bits are directed towards the bar port (upper port in the figure) because of the refractive-index change induced by beam. The physical mechanism behind the behavior is cross phase modulation
(XPM). Gain saturation induced by beam reduces carrier density inside one SOA, which in turn increases the refractive index only in the arm through which the light passes. As a result, an additional phase shift can be introduced on the CW beam because of the XPM, and the CW wave is directed towards the bar port during each one bit. [1-3]

Optical filters are placed in front of the output ports for blocking the original signal. The MZ scheme is preferable over cross gain saturation as it does not reverse the bit pattern and results in a higher on-off contrast simply because nothing exits from the bar port during 0 bit. Now it is clear that in the absence of control signal, the incoming signal (CW signal) exits through the cross port (lower channel) of MZI. In this case no light is present in the bar port as shown in the below

*Corresponding author:

V. Khanaa (drvkannan62@yahoo.com) 


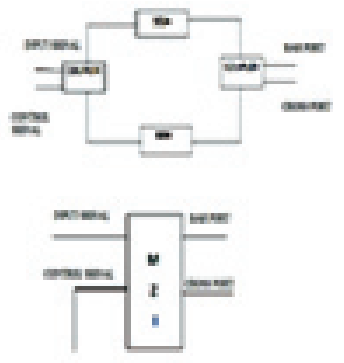

Figure 1. MZI Switch.

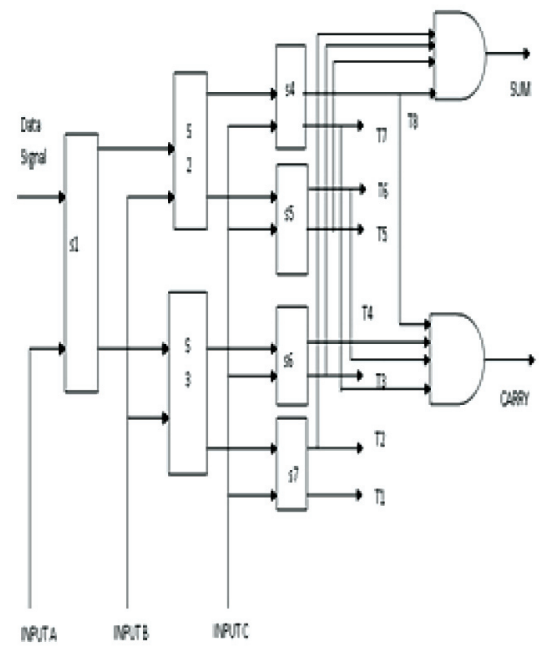

Figure 2. Full Adder block diagram.

figure. But in the presence of the control signal, the incoming signal exits through the bar port of the MZI as shown in the figure. In this case no light is present in the cross port. In the absence of the incoming signal, the bar port and cross port receive no light as the filter blocks the control signal. [4-5]

\section{Working of Full Adder}

To implement OTA for triple-in-binary logic taken another four MZI-based optical switches s4, s5, s6 and s7 as shown in Figure 3 In this architecture the continuous wave laser source falls on switch s1. For full adder three control signals is needed. Control signal A is given to the switch s1, control signal $\mathrm{B}$ is given to the switch $\mathrm{s} 2$ and $\mathrm{s} 3$ and control signal $\mathrm{C}$ is given to the switch $s 4, \mathrm{~s} 5, \mathrm{~s} 6$, and $\mathrm{s} 7$. When the control signal is presented then the light emerges upper channel and falls on the next channel and when the control signal is absent them light emerges from the lower channel followed by the next switch. There are eight different input combinations for implementing triple input binary logic. Depending on the state of input variables $(\mathrm{A}, \mathrm{B}, \mathrm{C})$ [these are also the light signals] the output is obtained from output terminal T-1 to output terminal T-8. Sum and carry are obtained by combining the corresponding terminals. The physical mechanism behind the behavior is crossphase modulation (XPM). Gain saturation induced by the 1 beam reduces carrier density inside one SOA, which in turn increases the refractive index only in the arm through which the 1 beam passes As a result, an additional phase shift can be introduced on the CW beam because of XPM, and the $\mathrm{CW}$ wave is directed towards the bar port during each one bit Optical filters are placed in front of the output ports for blocking the original signal. The MZI scheme is preferable over cross-gain saturation as it does not reverse the bit pattern and results in a higher on off contrast simply because nothing exits from the bar port during 0 bits are shown in the Figure 1.

The semiconductor optical amplifier (SOA) is of small size and electrically pumped. It can be potentially less expensive than the EDFA and can be integrated with semiconductor lasers, odulators etc. However, the performance is still not comparable with the EDFA. The SOA has higher noise, lower gain, moderate polarization dependence and high nonlinearity with fast transient time. This originates from the short nanosecond or less upper state lifetime, so that the gain reacts rapidly to changes of pump or signal power and the changes of gain also cause phase changes which can distort the signals. are shown in the Figure 2.

\section{Software Used}

Optsim is an advanced optical communication system simulation package designed for professional engineering and cutting-edge research of WDM, DWDM, TDM, optical LAN, parallel optical bus, and other emerging optical systems in telecom, data com, and other applications. It can be used to design optical communication systems and simulate them to determine their performance considering various component parameters. Optsim is designed to combine the greatest accuracy and modeling power with ease of use on both Windows and UNIX platforms. Optsim represents an optical communication system as an interconnected set of blocks, with each block representing a component or subsystem in the communication system. As physical signals are passed between components in a real world communication system. 


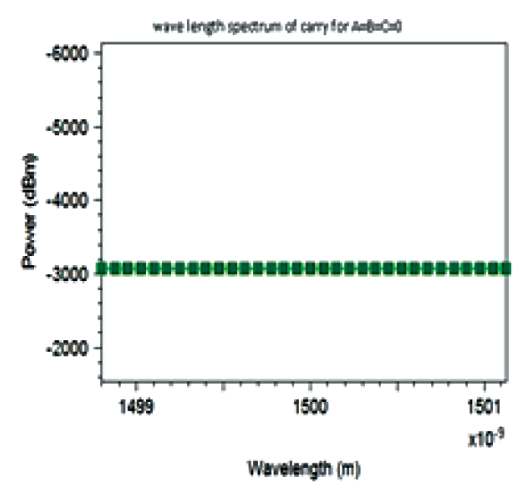

Figure 3. Wavelength spectrum for $\operatorname{sum}(\mathrm{A}=\mathrm{B}=\mathrm{C}=0)$.

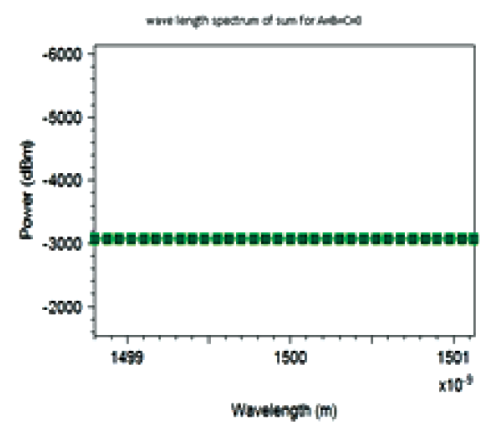

Figure 4. Wave length spectrum and carry $(\mathrm{A}=\mathrm{B}=\mathrm{C}=0)$.
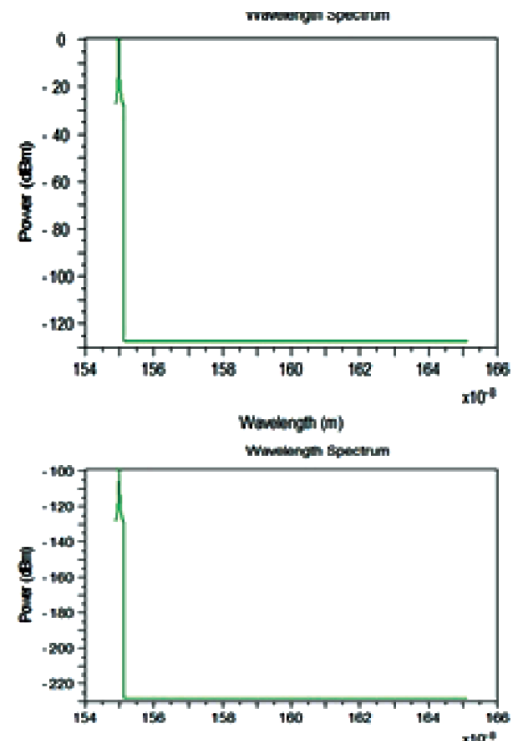

Figure 5. Wave length spectrum $S$ for sum and carry $(\mathrm{A}=\mathrm{B}=0, \mathrm{C}=1)$.
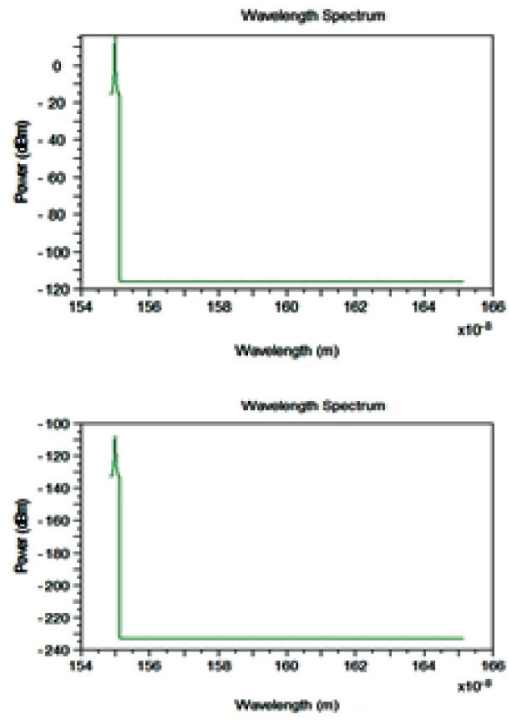

Figure 6. Wave length spectrums For Sum And Carry $(\mathrm{A}=\mathrm{C}=0, \mathrm{~B}=1)$.

\section{Results}

For 000 Logic state at the full adder input the sum and the carry output sare logicalzeros (Figure 4). Thus the wavelength spectrum for sum and carry show negative spectra. For 001 logical condition Sum is high and carry is low, spectrum will be positive forsum and negative for carry (Figure 5). For 010 logical condition sum is having a positive spectrum and for carry the spectrum is negative (Figure 6). Similarly all sum and carry outputs up to 111 logical conditions can be obtained.

\section{Conclusion}

An all optical full adder and de multiplexer using SOA based MZI switch is designed and output spectrums for the control signal values $A=B=C=0, A=B=0, C=1, A=0, B=1, C=0$ are obtained. This type of optical combinational circuits can be used for obtaining various high speed optical networks. By combining beam splitters, beam combiners, and cascading SOA-MZI switches, we can implement and realize any complex logical function expressed in sum of products form. This scheme can be easily and successfully extended for any higher number of input digits by proper incorporation of MZI based optical switches, vertical and horizontal extension of the tree and by suitable branch selection. 


\section{References}

1. Hill M T et al. (2005). Coupled Mach-Zehnder interferometer memory element, Optics Letters, vol 30(13), 1710-1712.

2. Mehra R, and Tripathi J K (2010). All optical switching in a semiconductor optical amplifier based Mach Zehnder interferometer, International Journal of Computer Application, vol 1(9), 102-106.
3. Li Z (2006). Ultrafast all optical signal processing using semiconductor optical amplifier, Journal of Light waves, vol 24(1), 230-236.

4. Poustie A (2007). Semiconductor optical amplifier lights up all optical signal processing, Journal of Lightwave Technology, vol 25(1), 103-108.

5. Huang D (2008). Potential optoelectronic devices for all optical signal processing, SPIE Proceedings, vol 7134. 